\title{
Behavioral changes in mice caused by Toxoplasma gondii invasion of brain
}

\author{
Justyna Gatkowska • Marek Wieczorek • \\ Bozena Dziadek • Katarzyna Dzitko • \\ Henryka Dlugonska
}

Received: 19 October 2011 / Accepted: 19 December 2011 /Published online: 6 January 2012

(C) The Author(s) 2012. This article is published with open access at Springerlink.com

\begin{abstract}
Toxoplasma gondii, a protozoan parasite, is capable of infecting a broad range of intermediate warm-blooded hosts including humans. The parasite undergoes sexual reproduction resulting in genetic variability only in the intestine of the definitive host (a member of the cat family). The parasite seems to be capable of altering the natural behavior of the host to favor its transmission in the environment. The aim of this study was to evaluate the number of parasite cysts formed in the hippocampus and amygdala of experimentally infected mice as these regions are involved in defense behaviors control and emotion processing, and to assess the influence of the infection on mice behavior. The obtained results revealed the presence of parasite cysts both in the hippocampus and the amygdala of infected mice; however, no clear region-dependent distribution was observed. Furthermore, infected mice showed significantly diminished exploratory activity described by climbing and rearing, smaller preference for the central, more exposed part of the OF arena and engaged in less grooming behavior compared to uninfected controls.
\end{abstract}

\section{Introduction}

Toxoplasmosis is one of the most common parasitic invasions worldwide, caused by the intracellular protozoan

J. Gatkowska $(\bowtie) \cdot$ B. Dziadek $\cdot$ K. Dzitko $\cdot$ H. Dlugonska

Department of Immunoparasitology, University of Lodz,

Banacha 12/16,

90-237 Lodz, Poland

e-mail: gatjus@biol.uni.lodz.pl

M. Wieczorek

Department of Neurophysiology, University of Lodz,

Pomorska 141/143,

90-236 Lodz, Poland parasite Toxoplasma gondii. The parasite is capable of infecting many species of warm-blooded animals and also humans serving as intermediate hosts (Elmore et al. 2010). The sexual reproduction occurs only in members of the cat family and results in generation of oocysts shed to the environment with the feces of infected cat. The oocysts may be ingested by wild rodents and other intermediate hosts leading to the asexual reproduction of $T$. gondii. The acute invasion, characterized by parasitemia, is a transient stage followed by chronic invasion when parasites reside within tissue cysts localized mainly in the central nervous system, muscle, and eye tissues (Innes 2010). The completion of $T$. gondii life cycle occurs when the cat consumes an infected intermediate host. Hence any behavior of an intermediate host that increases the chance of predation may lead to higher rate of parasite transmission to a cat. It is suggested that the presence of the parasite in the specified regions of the brain in rodents affects its physiological functions resulting in behavioral changes which are supposed to favor the spread of the parasite to the definite host (Felidae) (Webster 2001). Moreover, it was experimentally proven that behavioral changes are highly specific because Toxoplasma invasion blocks rodent aversion toward cat predator odor (Vyas et al. 2007; Berdoy et al. 2000) but does not affect recognition of the non-feline predator scent (Lamberton et al. 2008). Since the hippocampus and amygdala are brain structures connected with natural defense behaviors and emotion processing (da Silva and Langoni 2009), the aim of the study was to evaluate and compare the number of $T$. gondii cysts present in both brain regions and to assess the behavioral changes accompanying the parasite invasion of the brain. The experiments were performed on $\mathrm{C} 57 \mathrm{BL} / 6$ inbred mice, innately highly susceptible to toxoplasmosis, during both acute and chronic phases of $T$. gondii invasion. 


\section{Material and methods}

Animals

For the experiments male $\mathrm{C} 57 \mathrm{BL} / 6\left(\mathrm{H}-2^{\mathrm{b}}\right)$ inbred mice, $10-$ 12 weeks of age were used. The experimental groups were comprised of 6-14 animals. All experimental procedures were approved by the 9. Local Ethics Commission in Lodz.

Parasite

The intermediate virulent ME49 strain of T. gondii was used to induce the experimental toxoplasmosis in mice. The ME49 strain belongs to the group II of $T$. gondii highly cyst-forming strains most often associated with toxoplasmosis in humans, especially in Europe and North America. The parasite cysts were obtained from the brain of a chronically infected C57BL/6 mouse according to previously described protocol (Gatkowska et al. 2006). Briefly, the brain was mechanically homogenized and the cysts were recovered from mouse tissues by gradient separation. The obtained parasites were used to infect mice by intraperitoneal administration of 20 cysts per mouse.

Invasion parameters

The course of experimental toxoplasmosis was monitored by determination of brain, spleen, and body weight. These parameters were evaluated 3 weeks after primary infection which corresponds to the late acute invasion and 6 weeks after cyst administration, during chronic toxoplasmosis and compared to the results obtained in the group of noninfected mice of the same age and gender.

\section{Cyst burden determination}

The cysts were enumerated during late acute ( 3 weeks postinfection-p.i.) and chronic (6 weeks p.i.) toxoplasmosis in specified regions of the brain-hippocampus and amygdala in comparison to all other parts of the brain. Isolated fragments of brain tissues were placed into calibrated tubes, weighed and mechanically homogenized in phosphatebuffered saline to the final volume of 1 or $3 \mathrm{ml}$ (depending on the structure volume). The cysts were counted in duplicate samples of the homogenates by the use of light microscopy, in a volume of 50-100 $\mu$ l. The results were presented as the mean number of cysts per $1 \mathrm{mg}$ of respective brain fragment.

\section{Histology}

The brains of $T$. gondii-infected mice were dissected and fixed for $24 \mathrm{~h}$ in $4 \%$ buffered neutral formaldehyde.
Paraffin-embedded tissues were cut in 5- $\mu \mathrm{m}$ sections, stained with hematoxylin and eosin (H\&E), and examined by light microscopy.

\section{Behavioral test}

The behavior of non-infected and T. gondii acutely (3 weeks p.i.) and chronically (6 weeks p.i.) infected mice was quantified in an open field (OF) using a box $59 \times 59 \times 30 \mathrm{~cm}$ with its floor divided into center and peripheral parts which was illuminated with red light (20 lx at the floor level). Each mouse was gently placed in the center of the arena facing selected corner of the box, videotaped for $5 \mathrm{~min}$, and scored with the EthoVision system (ver. 2.3, Noldus, Wageningen, The Netherlands). The three groups of behavioral parameters were measured: (a) exploratory activity (rearing and climbing on the walls), (b) spontaneous locomotor activity (entries to the central and peripheral parts of the OF arena), and (c) anxiety-like behavior (self-grooming).

\section{Statistical analysis}

Statistical analysis of invasion parameters and brain cyst burden was performed with Mann-Whitney $U$ test. Differences were considered significant with $p<0.05$. All data obtained in OF test are reported as the mean \pm standard error of the mean. Analysis of variance (ANOVA) was performed using Statistica ver. 9.0 followed by Fisher's least significant difference (LSD) test. The three time groups, 0 , 3 , and 6 weeks after $T$. gondii inoculation and the central and peripheral parts of the OF arena, were used to determine main effects of Toxoplasma on mouse behavior.

\section{Results}

Invasion parameters and brain cyst burden assessment

The obtained results showed evident, statistically significant increase in brain $(p<0.020)$ and spleen $(p<0.004)$ weights during acute toxoplasmosis (Fig. 1a, b). Chronic phase was characterized by a significant decrease of organ weight to the values comparable with the uninfected controls $(p<$ 0.002 for brain and $p<0.004$ for spleen). The parasitic invasion was accompanied by a gradual decrease of body weight along with infection time. The body weight loss was statistically significant in acute toxoplasmosis $(p<0.002)$ as compared to the non-infected mice and in those with chronic phase $(p<0.020)$ in comparison to the acutely infected animals (Fig. 1c).

The next step was to assess the number of parasite cysts present in two structures of the brain implied in emotion processing and behavior control, namely hippocampus and 
Fig. 1 Brain (a), spleen (b), and body (c) weight of infected animals during $T$. gondii invasion

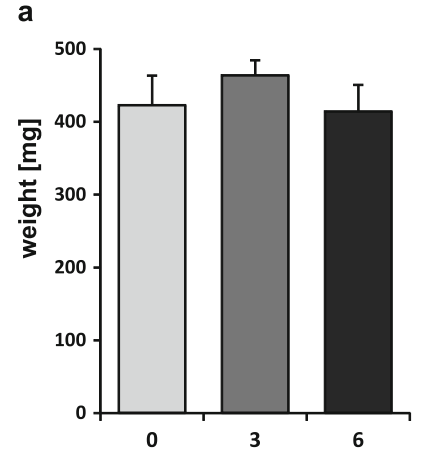

b

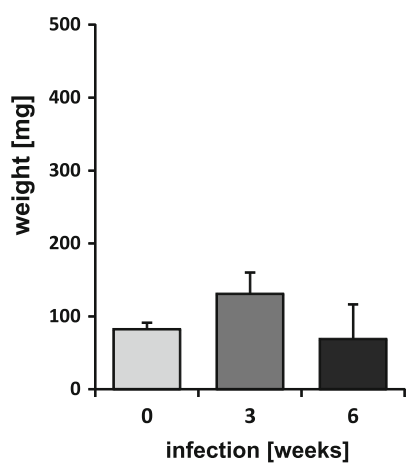

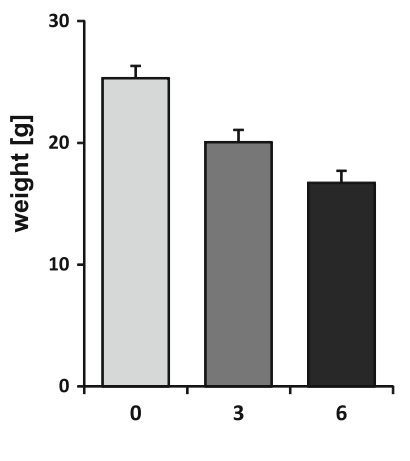

amygdala. As shown in Fig. 2, the cysts seem to be slightly more numerous in the hippocampus during acute parasite invasion and tend to be present in larger number in the amygdala during latent toxoplasmosis. However, the statistical analysis failed to prove observed differences significant due to great variability between individual results. The presence of parasite cysts in hippocampus and amygdala was also determined by the light microscopy of stained brain sections (Fig. 3).

\section{Activity in the open field}

Obtained results revealed that $T$. gondii invasion significantly influences the normal exploratory behavior of mice which was reflected by the statistically significant decrease in climbing during infection (ANOVA $F_{(2,29)}=69.77, p<0.001$ ) both in acute (LSD, $p<0.001$ ) and chronic (LSD, $p<0.001$ ) toxoplasmosis compared to uninfected controls (Fig. 4a). Another parameter connected with exploration - rearing, was also significantly altered by the parasite invasion. The ANOVA analysis revealed significant differences in number of rears in regard to the time after infection $\left(F_{(2,29)}=9.88, p<0.001\right)$, the part of OF arena $\left(F_{(1,29)}=289.45, p<0.001\right)$ and the interaction between time and part of the OF arena $\left(F_{(2,29)}=9.45, p\right.$

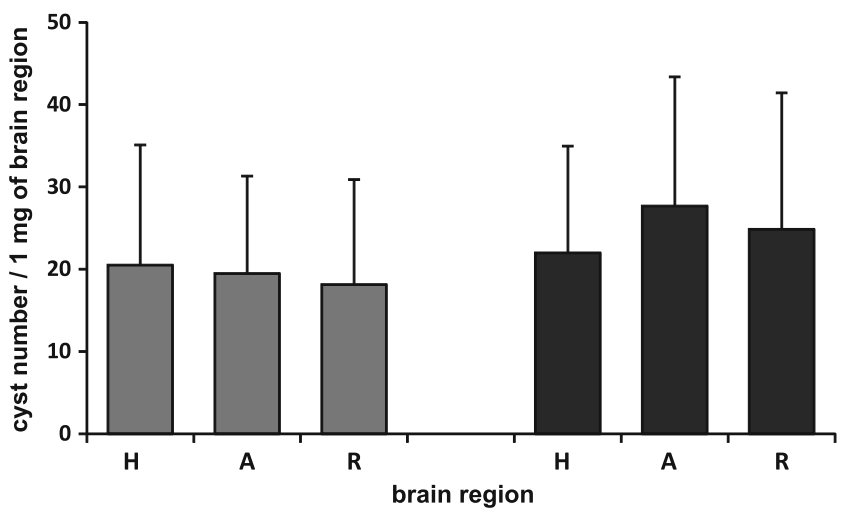

Fig. 2 The mean number of $T$. gondii cysts present in $1 \mathrm{mg}$ of specified brain region in the acutely (grey square) and chronically (black square) infected mice; $H$ hippocampus, $A$ amygdala, $R$ all other parts of the brain
$<0.001)$. As presented in Fig. 4b, infected mice exhibited a decrease in number of rears in the central part of the OF arena compared to uninfected animals during both acute (LSD $p=$ 0.046 ) and chronic (LSD $p=0.058$ ) toxoplasmosis, however, noticed differences were on the verge of significance. Statistically significant changes in the number of rears in the peripheral region of the OF were noticed only for the chronically infected mice (LSD, $p<0.001$ ).

Our findings proved that $T$. gondii also influences locomotor activity expressed by entries to the specified arena part (Fig. 5). Observed effect was statistically significant in relation to the invasion time $\left(F_{(2,29)}=10.58, p<0.001\right)$, the arena part $\left(F_{(1,29)}=134.11, p<0.001\right)$, and the interaction
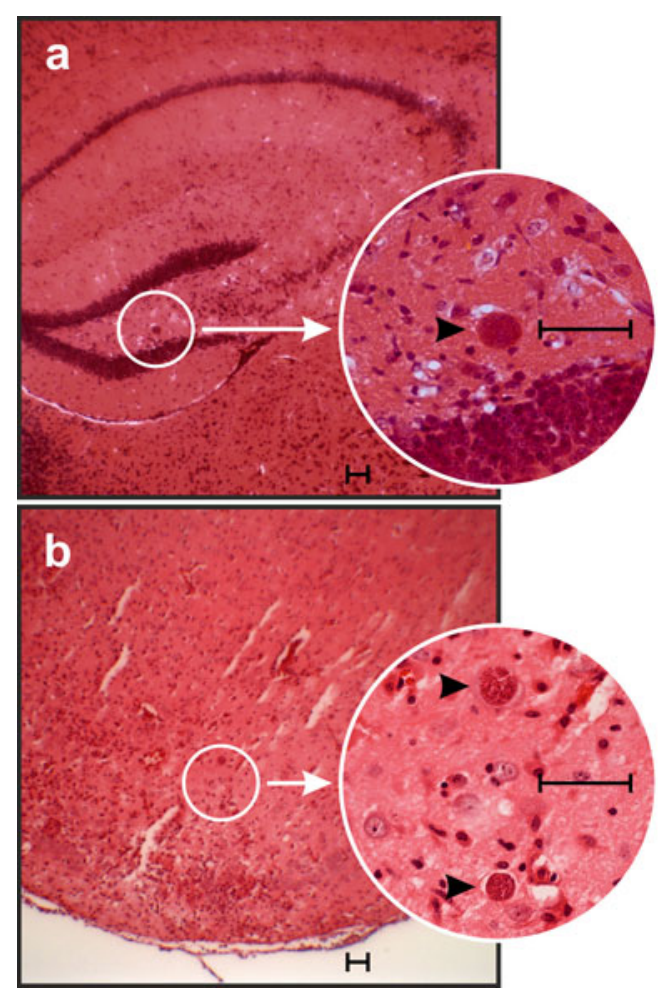

Fig. 3 Light micrographs of H\&E-stained brain sections from C57BL/ 6 mouse infected with ME49 T. gondii strain. The parasite cysts are present in hippocampus (a) and amygdala (b). Scale bar indicates $50 \mu \mathrm{m}$. Black arrows mark the $T$. gondii cysts 
Fig. 4 Changes in the exploration parameters: climbing (a) and rearing (b) of mice acutely and chronically infected with $T$. gondii in comparison to uninfected controls (time 0 )

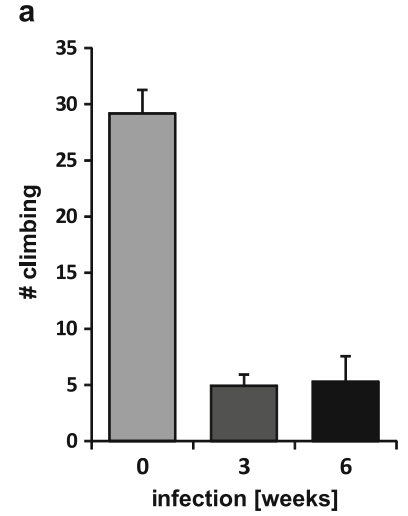

b

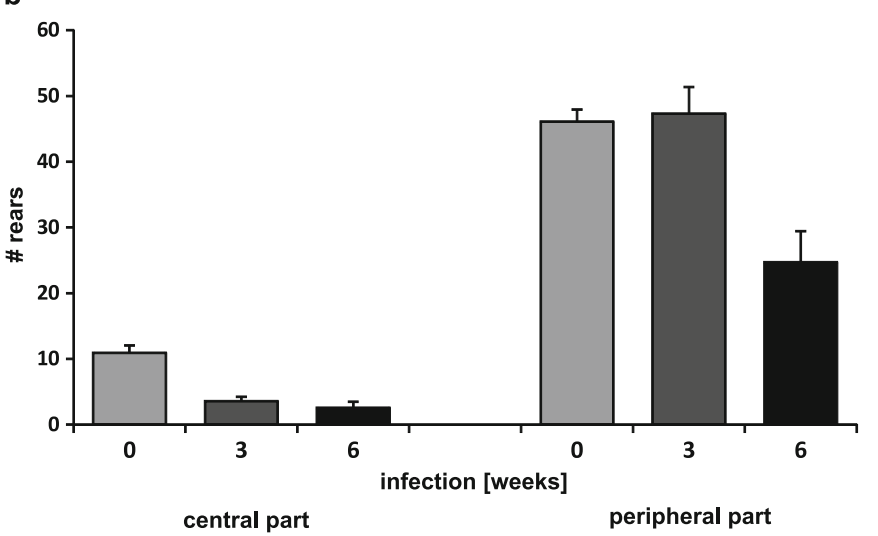

\section{Discussion}

It has been reported before that $T$. gondii invasion may alter the indefinite host natural defensive behavior in order to increase the risk of rodent predation by cats, the definite parasite hosts (da Silva and Langoni 2009; Holliman 1997). The main structures of the brain involved in defense behaviors (conditioned or learned fear) and nonconditioned anxiety are hippocampus and amygdala (da Silva and Langoni 2009) hence these structures were studied in relation to $T$. gondii invasion. In this paper, the presence of parasite cysts in two brain regions of interest was determined both in acute and chronic toxoplasmosis and the invasion process was further characterized by measurement of brain, spleen, and body weights. The results revealed increase in both brain and spleen weights during acute phase of $T$. gondii infection. Spleen, as the predominant secondary lymphoid organ, participates in the development of specific immunological response to parasite antigens, aimed at the elimination of the protozoan from the host organism. Intense accumulation of inflammatory cells is reflected by pronounced increase in spleen weight. The immunological response of the host

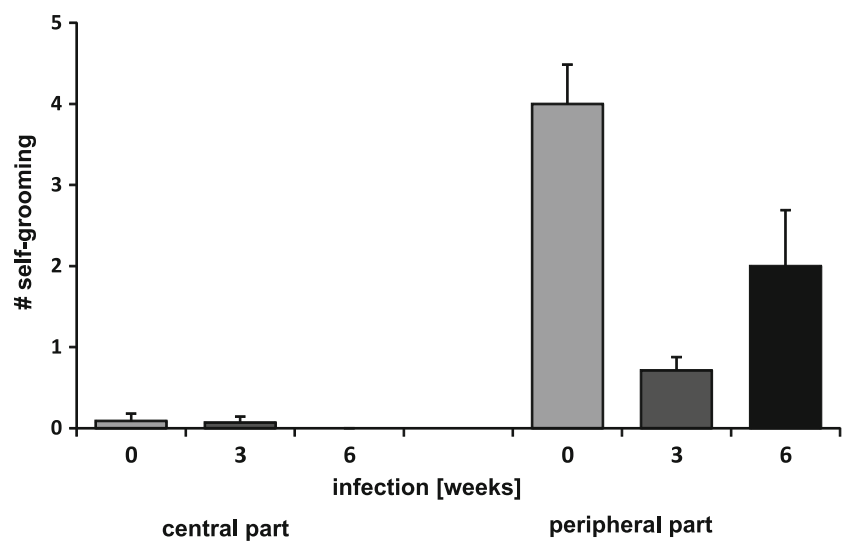

Fig. 6 Emotional status expressed by self-grooming of mice during acute and chronic toxoplasmosis 
contributes to the conversion of the rapidly dividing tachyzoites to bradyzoites which is accompanied by slow decrease of both inflammatory response and spleen weight in chronic toxoplasmosis. Also the transient increase in brain mass may be attributed to the inflammatory processes driven by many pro-inflammatory cytokines like IL-1 $\beta$ and IL-6, produced in brain during acute infection (Carruthers and Suzuki 2007) and accumulation of inflammatory cells (Ferguson and Hutchison 1987). The decrease in a brain weight, observed in chronic toxoplasmosis, to the measurements comparable with non-infected controls, may result simply from the repression of inflammatory reaction in brain. However, Hermes et al. (2008) reported the pronounced decrease of brain weight along with the prolonged (to about a year) parasite invasion although they failed to prove neuronal loss or demyelination.

The experiments were performed on the C57BL/6 mice, relatively more susceptible to $T$. gondii invasion and the gradual continued loss of body weight was observed. These mice are unable to properly extinguish the inflammatory response provoked by the parasite and as the result toxoplasmosis proves fatal, as described previously (Gatkowska et al. 2006). The development of progressive wasting syndrome was also reported in regard to other mouse strains (Stahl et al. 1994).

The analysis of the number of $T$. gondii cysts in specified regions of the brain failed to prove any preferences in occupancy probably due to differences between individual results. Experiments conducted by Vyas et al. (2007) on rat model showed that cyst density in amygdalar structures was twofold higher than in nonamygdalar structures. Noteworthy, the great spread of individual results, hindering the statistical analysis, was also noted. Other groups reported the presence of $T$. gondii cysts in selected regions of mouse brain, for instance Hermes et al. (2008) found T. gondii cysts in hippocampus of latently infected BALB/c mice, however, no comparison to amygdala was performed. Nevertheless, the presence of parasite in the structures of brain involved in conditioned or learned fear as defense behaviors and nonconditioned anxiety (da Silva and Langoni 2009) is undeniable. Hence OF test was performed and the results revealed changes in mice behavior after Toxoplasma challenge compared to uninfected controls. Infected animals exhibited decreased climbing and rearing especially in the central part of the arena. These behaviors reflect the explorative activity since they are the most basic means of gathering information about the environment used by nocturnal species with poor vision (Gulinello et al. 2010). Furthermore, all infected mice showed diminished preference to enter the central part of the arena. Normally, in unfamiliar surroundings mice first explore the peripheral parts and then they move to the central, open part of the arena (Holliman 1997). After Toxoplasma invasion animals tended to stay in the peripheral area of the
OF arena, especially during acute toxoplasmosis, which means that $T$. gondii infection inhibited the natural course of exploration. Similar findings related to exploration activity have been reported previously (Hay et al. 1983, 1984). These results comply with the manipulation theory according to which the parasite alters the behavior of the intermediate host to increase a chance of predation by the definitive host. The potential advantage for the parasite is obvious since only in the intestine of the definitive host the protozoan is able to complete the sexual stage of its life cycle which is necessary for the recombination resulting in genetic variability. Our finding also indicates increase in number of movements (data not shown), a phenomenon described previously (Hay et al. 1983, 1984, 1985), however, only during acute invasion and only in the peripheral part of the arena. This behavior may also favor predation since cats are mostly attracted to moving objects. Obtained results also revealed the influence of the parasite invasion of brain on animal emotional status. Infection drastically diminished the number of self-grooming episodes, mostly during acute infection phase, which is a typical "displacement activity" reflecting the approach-avoidance conflict (Hay et al. 1984). According to Dunn et al. (1987) and Dunn and File (1987) self-grooming may be considered an indicator of the emotional status correlating with the activity of the hypothalamus-pituitary-adrenal axis.

Noteworthy, in our experiments many of the observed behavioral changes were mostly pronounced during acute invasion. Hrdá et al. (2000) suggested that behavioral alterations may be transient, nonspecific byproducts of inflammatory reaction in the brain. Nevertheless there exists a strong body of evidence supporting the manipulation theory and indicating that $T$. gondii influence on the intermediate host is highly parasite specific (Vyas et al. 2007; Berdoy et al. 2000; Lamberton et al. 2008).

It is speculated how the parasite manages to alter the natural host behavior. Several literature data suggest possible mechanisms involved in the process, like disturbance in the level of neurotransmitters in the brain (Prandovszky et al. 2011; Skallová et al. 2006; Stibbs 1985), nevertheless the exact mechanism involved in host behavior manipulation remains unexplained. Furthermore, behavioral changes due to Toxoplasma invasion are not only reserved to rodents but also involve humans. Flegr (2007) reported pronounced differences in several personality features between Toxoplasmainfected and uninfected individuals, both men and women, hence further investigation is needed especially taking into consideration the possible link between $T$. gondii carrier-state, defined by the presence of specific antibodies and the elevated risk of developing serious disorders like schizophrenia (Torrey and Yolken 2003; Yolken et al. 2009), Parkinson disease (Miman et al. 2010), epilepsy (Stommel et al. 2001), or psychosis (Zhu 2009). 
Acknowledgments M.Sc. Magdalena Kostrzewa is thanked for her contribution to this study.

The study was supported by the Ministry of Science and Higher Education (grant N N302 636340) and University of Lodz (grant 545/211).

Open Access This article is distributed under the terms of the Creative Commons Attribution Noncommercial License which permits any noncommercial use, distribution, and reproduction in any medium, provided the original author(s) and source are credited.

\section{References}

Berdoy M, Webster JP, Macdonald DW (2000) Fatal attraction in rats infected with Toxoplasma gondii. Proc Biol Sci 267:1591-1594

Carruthers VB, Suzuki Y (2007) Effects of Toxoplasma gondii infection on the brain. Schizophr Bull 33:745-751

da Silva RC, Langoni H (2009) Toxoplasma gondii: host-parasite interaction and behavior manipulation. Parasitol Res 105:893898

Dunn AJ, File SE (1987) Corticotropin-releasing factor has an anxiogenic action in the social interaction test. Horm Behav 21:193202

Dunn AJ, Berridge CW, Lai YI, Yachabach TL (1987) CRF-induced excessive grooming behavior in rats and mice. Peptides 8:841844

Elmore SA, Jones JL, Conrad PA, Patton S, Lindsay DS, Dubey JP (2010) Toxoplasma gondii: epidemiology, feline clinical aspects, and prevention. Trends Parasitol 26:190-196

Ferguson DJ, Hutchison WM (1987) An ultrastructural study of the early development and tissue cyst formation of Toxoplasma gondii in the brains of mice. Parasitol Res 73:483-491

Flegr J (2007) Effects of toxoplasma on human behavior. Schizophr Bull 33:757-760

Gatkowska J, Hiszczynska-Sawicka E, Kur J, Holec L, Dlugonska H (2006) Toxoplasma gondii: an evaluation of diagnostic value of recombinant antigens in a murine model. Exp Parasitol 114:220-227

Gulinello M, Acquarone M, Kim JH, Spray DC, Barbosa HS, Sellers R, Tanowitz HB, Weiss LM (2010) Acquired infection with Toxoplasma gondii in adult mice results in sensorimotor deficits but normal cognitive behavior despite widespread brain pathology. Microbes Infect 12:528-537

Hay J, Hutchison WM, Aitken PP, Graham DI (1983) The effect of congenital and adult-acquired Toxoplasma infections on activity and responsiveness to novel stimulation in mice. Ann Trop Med Parasitol 77:483-495

Hay J, Aitken PP, Graham DI (1984) Toxoplasma infection and response to novelty in mice. Parasitol Res 70:575-588
Hay J, Aitken PP, Arnott MA (1985) The influence of congenital Toxoplasma infection on the spontaneous running activity of mice. Z Parasitenkd 71:459-462

Hermes G, Ajioka JW, Kelly KA, Mui E, Roberts F, Kasza K, Mayr T, Kirisits MJ, Wollmann R, Ferguson DJ, Roberts CW, Hwang JH, Trendler T, Kennan RP, Suzuki Y, Reardon C, Hickey WF, Chen L, McLeod R (2008) Neurological and behavioral abnormalities, ventricular dilatation, altered cellular functions, inflammation, and neuronal injury in brains of mice due to common, persistent, parasitic infection. J Neuroinflammation 5:48

Holliman RE (1997) Toxoplasmosis, behaviour and personality. J Infect 35:105-110

Hrdá S, Votýpka J, Kodym P, Flegr J (2000) Transient nature of Toxoplasma gondii-induced behavioral changes in mice. J Parasitol 86:657-663

Innes EA (2010) A brief history and overview of Toxoplasma gondii. Zoo Pub Health 57:1-7

Lamberton PH, Donnelly CA, Webster JP (2008) Specificity of the Toxoplasma gondii-altered behaviour to definitive versus nondefinitive host predation risk. Parasitology 135:1143-1150

Miman O, Kusbeci OY, Aktepe OC, Cetinkaya Z (2010) The probable relation between Toxoplasma gondii and Parkinson's disease. Neurosci Lett 475:129-131

Prandovszky E, Gaskell E, Martin H, Dubey JP, Webster JP, McConkey GA (2011) The neurotropic parasite Toxoplasma gondii increases dopamine metabolism. PLoS One 6:e23866

Skallová A, Kodym P, Frynta D, Flegr J (2006) The role of dopamine in Toxoplasma-induced behavioural alterations in mice: an ethological and ethopharmacological study. Parasitology 133:525-535

Stahl W, Kaneda Y, Noguchi T (1994) Reproductive failure in mice chronically infected with Toxoplasma gondii. Parasitol Res 80:22-28

Stibbs HH (1985) Changes in brain concentrations of catecholamines and indoleamines in Toxoplasma gondii infected mice. Ann Trop Med Parasitol 79:153-157

Stommel EW, Seguin R, Thadani VM, Schwartzman JD, Gilbert K, Ryan KA, Tosteson TD, Kasper LH (2001) Cryptogenic epilepsy: an infectious etiology? Epilepsia 42:436-438

Torrey EF, Yolken RH (2003) Toxoplasma gondii and schizophrenia. Emerg Infect Dis 9:1375-1380

Vyas A, Kim SK, Giacomini N, Boothroyd JC, Sapolsky RM (2007) Behavioral changes induced by Toxoplasma infection of rodents are highly specific to aversion of cat odors. Proc Natl Acad Sci U S A 104:6442-6447

Webster JP (2001) Rats, cats, people and parasites: the impact of latent toxoplasmosis on behaviour. Microbes Infect 3:1037-1045

Yolken RH, Dickerson FB, Fuller Torrey E (2009) Toxoplasma and schizophrenia. Parasite Immunol 31:706-715

Zhu S (2009) Psychosis may be associated with toxoplasmosis. Med Hypotheses 73:799-801 